\title{
The effects of stimulus variability on response latency in a continuous recognition task
}

\author{
DONALD S. CICCONE, JOHN W. BRELSFORD, and THOMAS TULLIS \\ Rice University, Houston, Texas 77001
}

\begin{abstract}
Using a test-study recognition task, stimulus variability was manipulated by pairing a given critical noun with either one or four different adjectives on successive presentations. This defined two levels of variability, low and high, respectively. Yes-No recognition latencies to the fifth presentation of critical items indicated that low variability items were responded to more rapidly than high variability items. This finding was discussed in terms of its implications for an encoding variability interpretation of context effects in recognition memory.
\end{abstract}

The issue we wish to consider concerns the nature of the retrieval process in recognition memory. A dual process theory of recall and recognition with separate retrieval operations for each has been suggested by Kintsch (1970) and others, who assign a minimal role to the retrieval process in recognition. Tulving and Thompson (1971), on the other hand, have argued that recognition memory can and does involve a complex retrieval (search) process. We take the position that the retrieval process in recognition, while possibly different from that in recall, can nevertheless involve "search".

In view of the recent findings involving contextual manipulations (e.g., Light and Carter-Sobell, 1970), it no longer seems possible to insist upon a "direct access" interpretation of retrieval in recognition. However, as Kintsch (1970), has pointed out, there exist numerous empirical findings which strongly suggest that recall and recognition involve different processes. Estes and DaPolito (1967) report, for example, that intentional learning instructions facilitate recall, but not recognition, performance. On the other hand, some experimental manipulations appear to have similar effects irrespective of test mode. Recent studies suggest, for example, that variability in the contextual environment of critical items improves recall (D'Agostino \& DeRemer, 1973) as well as recognition (Winograd \& Raines, 1972) performance. The concept of encoding variability has been invoked to account for these results in terms of an increased number of retrieval routes for items presented in variable as opposed to uniform contextual settings.

It would seem that this explanation of the contextual variability effect presumes a search process underlying retrieval in both recognition and recall. While this seems a safe assumption in the case of recall, a search process in recognition requires corroboration. The present study is concerned with providing some relevant data in this regard.

This research was supported in part by Public Health Service Research Grant MH22235-01 to the second author. Requests for reprints should be addressed to John W. Brelsford, Department of Psy chology, Rice University, Houston, Texas 77001 .
The encoding variability account of context effects in recognition (e.g., Winograd \& Raines, 1972) implies a search process during retrieval. If this is, in fact, the case then it seems reasonable to assume that high and low amounts of stimulus variability ought to produce differential response latencies in a simple yes-no recognition procedure. This prediction derives from the notion that a multiplexing of the stimulus trace enhances item accessibility by increasing the number of possible retrieval routes. The implicit assumption is that a single access route is sufficient to produce successful retrieval. Given that a search process operates in recognition, items presented in highly variable contexts should be responded to faster than items presented under more uniform circumstances.

In the present study we adopted the convention of defining stimulus variability in terms of the number of different adjectives a given critical noun was paired with. During the course of the experimental session, a particular critical noun might be paired with either one or four different adjectives, defining two levels of stimulus variability (low and high). Stimulus variability, as thus defined, may serve to render items either more or less accessible depending on the degree of variability present during input.

\section{METHOD}

\section{Experimental design and materials}

A modified Shepard and Teghtsoonian (1961) continuous recognition paradigm was used. Each trial consisted of a test phase, in which a noun was presented alone, followed immediately by a study phase in which the test item was paired with an adjective. The number of different adjective-noun pairings during the study phase of each trial, for a given item, defined two levels of stimulus variability. A given noun was paired with either one or four different adjectives during the study phase of successive presentations. High-frequency concrete nouns (Thorndike \& Lorge, 1944) served as the test words for all trials and were (within certain constraints) randomly paired with high frequency adjectives during the study phase of each trial. Bizarre and common pairings (e.g. ELECTRIC MOUTH and GREEN GRASS) were excluded. Each of 20 critical nouns was assigned a set of four adjectives with which it could be paired. 
This was necessary since each noun occurred at both levels of variability (across experimental lists). One of the four adjectives was chosen at random when a critical noun was assigned to the low variability condition.

There were two experimental lists. Each was computer generated and had the following characteristics: (1) each began with a 20 -item primacy buffer; (2) each was approximately 200 items in length; (3) each contained 10 critical items at each level of stimulus variability; (4) each critical item was presented exactly five times at each level of variability with 15 items intervening between successive repetitions; (5) on any trial the probability was .5 that the subject had not seen the item previously (buffer items were used to fulfill the requirements of 4 and 5). The same nouns and adjectives were used to construct both lists. The 20 critical test nouns were partitioned at random into two groups of 10 , with each group occurring at both levels of variability. Within each list the placement of the variability levels was block randomized, with each level occurring twice in each block of four consecutive critical items. A single list was administered to each subject.

\section{Procedure}

The duration of the test phase of each trial was determined by a subject's response latency, being terminated as soon as he responded. A 3-sec study phase involving an adjective-noun pair followed immediately. Stimuli were presented visually via a closed-circuit television display with $1 \mathrm{sec}$ intervening between trials. Each subject was instructed to rehearse overtly each adjective-noun pair exactly twice during the study phase of each trial. A keyboard, attached to a computer, was placed in front of the display. The numbers " 5 " and " 6 " were relabeled "no" and "yes" respectively. The subject was instructed to respond by pressing one of these two response keys as quickly as possible following the appearance of the test item. He was told to press "yes" only when the item was previously presented and to press "no" otherwise. These instructions intentionally imposed a yery rigid criterion for "yes" responses in order to minimize false alarms. The computer recorded the "yes-no" as well as the response latency on each of the five trials given to each of the 10 critical items at each level of variability. The false alarm rate as a function of trials was recorded by dividing each experimental session into fifths and determining the total number of "yes" responses to buffer items, as well as to first presentations of critical items. The total latency in milliseconds for these false alarms, in each fifth, was also recorded. Similar data were obtained for correct rejections, also partitioned into fifths.

\section{Subjects}

The subjects were 40 students at Rice University who volunteered to participate. They were assigned in block randomized fashion to one of two experimental lists $(20$ subjects per list).

\section{RESULTS}

Accuracy of recognition performance was essentially perfect with respect to critical items at both levels of variability. Since there were 10 critical items per subject at each level, each subject's latency score was a mean based on 10 observations. The mean (across subjects) response latency on the fifth presentation of critical items within each of the two experimental lists (at each level of variability) exhibited a consistent pattern of increased latency for high as opposed to low variability items. The data from both lists were therefore combined. The mean (across all subjects) response latency on the fifth presentation of critical items was
$602 \mathrm{msec}$ for low and $698 \mathrm{msec}$ for high variability items. A test for correlated measures indicated that this difference was significant, $[\mathrm{t}(39)=2.98, \quad \mathrm{p}<.01$ (two-tailed)].

The false alarm data, as a function of trials, was obtained by dividing each experimental list into fifths. The percentages of false alarm responses in each fifth were $3.3 \%, 3.0 \%, 4.2 \%, 3.3 \%$, and $4.4 \%$ consecutively. The mean latency of a false alarm was $806 \mathrm{msec}$ as compared to a mean value of $735 \mathrm{msec}$ for a correct rejection. A test for correlated observations was significant $[\mathrm{t}(39)=2.88, \mathrm{p}<.01$ (two-tailed) $]$.

\section{DISCUSSION}

Since each subject's "yes-no" recognition performance was nearly perfect on all trials at both levels of stimulus variability, it is reasonable to assume that all critical items were equally "available" (Tulving \& Pearlstone, 1966). If we interpret the latency of recognition response as an index of accessibility, then the present results suggest that accessibility in recognition is inversely related to increasing stimulus variability (as we have defined it). This result is contrary to what might be expected on the basis of encoding variability theory and is in at least nominal conflict with studies demonstrating improvement in performance with increases in contextual variability (e.g., Winograd \& Raines, 1972).

The encoding variability interpretation of context effects in recognition seems to imply that (a) search processes operate in recognition and (b) high variability items should be accessed more rapidly than low variability items. The finding of differential latencies across the two levels of variability in the present study secms to indicate that (a) is a reasonable assumption while the pattern of differences suggests that (b) is not. In choosing between "search" and "direct access" retrieval mudels, the finding of latency differences between false alarms and correct rejections, such as those obtained in the present study, are equivocal in the sense that they are consistent with both interpretations. However, the pattern of latency differences to the high and low variability items does seem to cast doubt on the adequacy of an encoding variability interpretation of context effects in recognition memory.

It may be the case that a simple "strength" concept is a more appropriate analog in describing the effects of stimulus variability upon recognition latency. The present results seem to contradict the notion that retrieval in recognition is positively correlated with the number of retrieval routes to critical items. The high variability items, associated with the higher number of access routes, were retrieved more slowly than the low variability items. A strength theory which places primary emphasis upon sheer number of nominal stimulus presentations would seem to be consistent with the present finding of lower latency to low variability items (which were presented in identical fashion on successive trials). This rationale, however, while consistent with the results of the present study, does not seem plausible given the Winograd and Raines (1972) finding that variably encoded items were recognized better than those presented in a uniform context. A recent model of recognition memory proposed by Atkinson and Juola (1974) seems to be reasonably consistent with both sets of findings. The model describes the retrieval process in recognition as involving either a "direct access" or a "search" process depending on the familiarity value of the test item. In terms of their model, the high variability items of the present study may have been subjected to a search process while the low variability items were of sufficient strength (familiarity) so as to be accessed directly, resulting in lower response latencies. 


\section{REFERENCES}

Atkinson, R. C., \& Juola, J. F. Search and decision processes in recognition memory. In D. A. Krantz, R. C. Atkinson, R. D. Luce, \& P. Suppes (Eds.), Learning, memory, and thinking (Vol. 1). San Francisco: Freeman, 1974. D'Agostino, P. R., \& DeRemer, P. Repetition effects as a Verbal Learning and Verbal Behavior, 1973,12, 108-113.

Estes, W. K., \& DaPolito, F. Independent variation of information storage and retrieval processes in paired-associate learning. Journal of Experimental Psychology, 1967, 75, 18-26.

Kintsch, W. Models for free recall and recognition. In D. A. Norman (Ed.), Models of human memory. New York: Academic Press, 1970.

Light, L. L., \& Carter-Sobell, L. Effects of changed semantic context on recognition memory. Journal of Verbal Leaming and Verbal Behavior, 1970,9,1-11.
Shepard, R. N., \& Teghtsoonian, M. Retention of information under conditions approaching a steady state. Journal of Experimental Psy chology, 1961, 62, 302-309.

Thorndike, E. L., \& Lorge, I. The teacher's word book of 30,000 words. New York: Teachers College, Columbia University Bureau of Publications, 1944

Tulving, E., \& Pearlstone, Z. A vailability versus accessibility of information in memory for words. Journal of Verbal Learning and Verbal Beh avior, $1966,5,381-391$.

Tulving, E., \& Thomson, D. M. Retrieval processes in recognition memory: Effects of associative context. Journal of Experimental Psy chology, 1971, 87, 116-124.

Winograd, E., \& Raines, S. R. Semantic and temporal variation in recognition memory. Journal of Verbal Learning and Verbal Behavior, 1972, 11, 114-119.

(Received February 6, 1975.) 